\title{
Zur urartäischen Nominalflexion
}

\author{
Von G. Wilhelm - Saarbrücken
}

In einer seiner für die Erforschung der urartäischen Grammatik grundlegenden Arbeiten ${ }^{1}$ hat J. Friedrich I93I ein Zugehörigkeitssuffix /ini/ segmentiert ${ }^{2}$, mit dem seiner Auffassung nach Kasus/ Numerus-kongruent konstruierte Adjektive gebildet werden. So werde von dem Namen des urartäischen Hauptgottes Haldi eine Form haldi=ini $=$ deriviert, die etwa ,dem Haldi gehörig" zu übersetzen sei $^{3}$. Dasselbe Morphem ist einem Hinweis Friedrichs zufolge ${ }^{4}$ bereits von Götze ${ }^{5}$ und Sayce ${ }^{6}$ in der Form /nil angesetzt worden. Ob /inil mit dem von ihm in demselben Artikel behandelten /hini/, das in Patronymika ${ }^{7}$ und Toponymika ${ }^{8}$ auftritt, identisch sei, läßt Friedrich dahingestellt sein?

Dieses Suffix / ini/ ist seitdem in die meisten grammatischen Darstellungen des Urartäischen aufgenommen worden ${ }^{10}$. D'jakonov ${ }^{11}$ identifiziert es mit einem hurritischen Morphem /inne/ (mit anderem Themavokal /onne/, |anne/|12 ${ }^{12}$ das im Hurritischen besonders Berufs-

1 J. Friedrich, Caucasica 8 (I93I) r I4 - I $_{50}$.

21.c. 127 .

${ }^{4}$ 1.c. 127 n. 5 .

${ }^{3}$ 1.c. 128.

${ }^{6}$ A. H. Sayce, JRAS I882, 434.

5 A. Götze, ZA 39 (1930) I I 2.

${ }^{7}$ z.B. menuaše išpuiniḩiniše, ,Menua, der Sohn des Išpuini“"; J. Friedrich, 1.c. 126 .

${ }^{8}$ z.B. argištiḩinili ,Argišti-Stadt"; $1 . c$.

${ }^{9}$ 1.c. 127 n. 5 .

${ }^{10}$ Friedrich, Einführung 9; I. T. Meščaninov, Grammatičeskij stroj urartskogo jazylka I, Moskva 1958, 87; Friedrich, Urartäisch 38, § I8b; Melikišvili, US 32; Diakonoff, $\mathrm{HuU} 7 \mathrm{O}$.

Skeptisch dagegen äußern sich M. de Tseretheli, RA 3I (I934) 42; Benedict, UPM I24-I32.

11 Diakonoff, $\mathrm{HuH} 70$ sub 3).

12 Der $e$-Vokal ist nur für den Artikel sg./ne/, doch nicht für das infragestehende Morphem nachgewiesen, da es im Mit. keine plene-Schreibung gibt. Eine neuere Studie zu dem Morphem stammt von F. W. Bush (The Relationship between the Hurrian Suffixes -ne/-na and -nni/e/-nna, in: Orient und Occident [Fs. C. H. Gordon], AOAT 22, Kevelaer/Neukirchen-Vluyn 1973, $39-52)$. 
bezeichnungen bildet ${ }^{13}$. Das Suffix sei im Urartäischen sehr produktiv, es könne jedes beliebige Nomen adjektivieren und stehe mit dem Genitivmorphem in freier Varianz ${ }^{14}$.

Die letztere Aussage impliziert, daß Sätze des Typs

(I) *haldi=ini susi tini $(=i y)^{15}$ "Tempel des Haldi ist sein Name" und

${ }^{13}$ E. von Schuler, RHA fasc. 68 (I96I) I9-23; Bush, GHL 274sq. Das Suffix /nni/ ist fälschlich mit dem. Artikel sg. /ne/ identifiziert worden, cf. E. A. Speiser, Introduction to Hurrian, AASOR 20, New Haven I94I, §§ 86a, 89, I37, I59; A. Kammenhuber, Hurrische Nomina, in: Studien zur Sprachwissenschaft und Kulturkunde, Gedenkschrift für Wilhelm Brandenstein, Innsbrucker Beiträge zur Kulturwissenschaft 14, Innsbruck 1968, 248sq., 257; eadem; MSS 23 (r968) 49sqq., 58; eadem, UF 2 (I970) 301; E. Laroche, Ugaritica V, Paris I968, 530.

Häufig tritt /nni/ in dem nomina actoris bildenden Komplex /kkonni/ auf, in $\operatorname{dem} \mid k k V /$ zumindest in dem häufigen aşhozikkkonni „Opferherr“" nicht seine sonst übliche negative Bedeutung hat; gegen die Skepsis von A. Kammenhuber, UF 2 (1970) 302, das Ugarit-hurritische ašlm = ugarit. $d b h$ "Opfer" mit E. Laroche, Ugaritica V, Paris 1968, 50I, als Infinitiv $a \bar{s} h u / o$ lumme $\mathrm{zu}$ vokalisieren und davon ausgehend $a \bar{s} h=o \bar{z}=i=k k o n n i \mathrm{zu}$ analysieren, spricht die urart. Verbalwurzel ašh̆- ,opfern" (HchI ı75; UKN 39osq.; Diakonoff, HuU 76). Das Urartäische kennt gleichfalls ein nichtnegatives Formans $/ k(k) /$ in nomina actoris; bisher einziges Beispiel ist LƯ urbikani ,,Schlachtopferpriester(?)" (zu urb-, ,schlachten").

F. W. Bush, AOAT 22, Kevelaer/Neukirchen-Vluyn 1973, 39-52, trennt zu Recht scharf zwischen dem Artikel /ne/ und einem , associative - $n n i^{\prime \prime}$, dem er adjektivierende Bedeutung ähnlich wie /ǵe/ sowie möglicherweise eine ,Partizip"-bildende Funktion zuerkennt.

14 Diakonoff, $\mathrm{HuU} 7 \mathbf{I}$ n. 72 ad (3); I03. Die an letzterer Stelle gegebenen Belege für freie Varianz von /ini/ und /i/ können nicht überzeugen; es handelt sich ausschließlich um nur hier belegte Personennamen mit nur teilweise klarer Morphologie, die alle auch als Genitive gedeutet werden können.

Einige der Namen enthalten das Element /iu/, das Diakonoff, $\mathrm{HuU}$ г8 und 68 vor allem in transkaukasischen Orts- und Stammesnamen sowie in drei urartäischen Berufsbezeichnungen findet. Daß es dazu keine Parallele im Hurritischen gibt (Diakonoff, $\mathrm{HuU}_{\mathrm{I}} 8$ ), ist nicht richtig; hier wäre eine groBe Zahl bisher ungedeuteter Personennamen aus Nuzi anzuführen: Akiu, Ariwaltiu, Atiu, Entiu, Haniu, Hašiu, Ikkiu, Kaniu, Kariu, Kikkiu, Kuziu, Mašiu, Metkiu, Natiu, Paltukkiu, Peliu, Walinniu, Zipiu, Zikiu.

15 Diakonoff, HuU Io9, setzt als Possessivsuffix der 3. Ps. -(i)'ja- (vor weiteren Enklitika unter Akzent) $\sim-(i) j($ (a) (unbetont in Endstellung) an. Das erstere Allomorph ist gesichert durch die Form $u l$-gu-ši-i-a-ni e-[di-ni] = akkad.

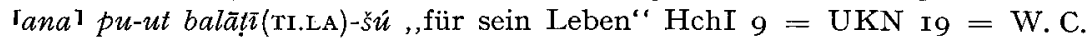
Benedict, JAOS 8I (I96r) 372//362:13//I2. Der Nachweis des zweiten Allomorphs ist schwieriger, da es zumeist, wie im vorliegenden Falle, auf -ifolgt. Der Ansatz ist aber gesichert durch die syllabische Entsprechung

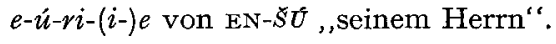


(2) haldi=i susi tini(=iy) in derselben Bedeutung

oder

(3) umešini tini ... rusa $=i n i=i$ h̆ubi=i , Umešini ist der Name des ... und Tales(?) des Rusa"

(4) *umešini tini . . rusa $=i \not h u b i=i$ in derselben Bedeutung nebeneinander stehen.

Dies ist aber offensichtlich nicht der Fall. Im folgenden sei ein repräsentativer Ausschnitt aus den in verständlichem Kontext belegten Formen des Genitivs sg. gegeben ${ }^{16}$ :

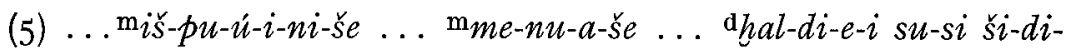
$i \check{\text { št } t u-n i}, \ldots$ Išpuini $\ldots$ (und) Menua haben (Text: sg.) einen Tempel des Haldi errichtet." HchI $8 \mathrm{I}=\mathrm{UKN}$ 25:I-2// 6 -7.

(6) ... dhal-di-i $i$-a-ra-ni ši-di-iš-tú-ni ,... einen Kultsockel ${ }^{17}$ des Haldi hat er errichtet." HchI 25 IV = UKN 39:I3-I4.

(7) $\mathrm{m}_{m e-n u-a-i}$ pi-li ti-i-ni , 'Kanal des Menua' ist (sein) Name." HchI $29 \mathrm{a}-\mathrm{d}$ I $=$ UKN $43: 3$.

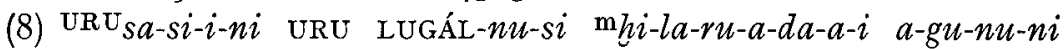
ma-nu ,Sasi, die Königsstadt des Hilaruada, war befestigt." HchI I04 VII $=$ UKN I58: I9-20.

(9) $[t] e-r u-b i$ ti-ni $\mathbf{m}_{r u-[s] a-a-i} s u-\Gamma_{e} \mathbf{l}$,,Ich setzte (seinen) Namen 'See des Rusa'." HchI I2I I = UKN 268:4.

Das Suffix /ini/ glaubte Friedrich zuerst in folgendem Satz feststellen zu können:

(Iо) $\mathrm{m}_{m e-n u-a-i-n i-e-i} \mathrm{SAL}_{s i-l a-a-(i-) e} \mathrm{f}_{t a-r i-r i-a-i} i-n i$ GIš $u l-d i \mathrm{HchI}$ $40=\mathrm{UKN}$ III:I-2//4-5.

Friedrich segmentierte memua=ini=ei und übersetzte: „Diese Weinpflanzung (ist die) der Menuaischen Gattin(?) ${ }^{18}$ Tariria."

${ }^{16}$ Zahlreiche weitere Belege finden sich bei J. Friedrich, Caucasica 8 (I93I) I I5-II7; M. de Tseretheli, RA 32 (1935) 36sqq.; Benedict, UPM I56-r6r.

17 iarani ist in der Kelišin-Bilingue mit akkad. par( $r) a k k u$ geglichen, das aB ein Heiligtum (, Kapelle"), in der jüngeren Sprache einen Kultsockel bezeichnet (cf. A. Schott, ZA 40 (I93I) I9-23); bei zahlreichen jüngeren Belegen ist es nicht klar, ob nicht doch das Heiligtum als ganzes oder die Cella gemeint ist (cf. AHw 828).

${ }^{18}$ sila entspricht hurr. sala und heißt demnach "Tochter"; cf. J. Friedrich, Kleine Beiträge zur Churritischen Grammatik, MVAeG 42/2, Leipzig 1939, 60; Friedrich, Urartäisch 47; UKN $4 \mathrm{O}_{4}$; Diakonoff, $\mathrm{HuU} 77$. Die Bedeutung ist nicht übernommen worden von Benedict, UPM I55 (,,wife(?)“). 
Im folgenden seien einige weitere Beispiele für Stellen geboten, an denen Friedrich dieses Suffix gefunden zu haben meinte:

(II) [d] hal-di-ni-e ba-du-si-e DUB-t[e te-r] $u$-í-bi HchI 74 Rs. II 3-4 $=$ UKN 99 obor. $2-3$.

Friedrichs Übersetzung faßt $b a-d u$-si-e als Attribut zu DUB-te auf und übersetzt: ,Die dem Haldi gehörige verfallene Tafel habe ich (wieder) aufgestellt." $b a$-du-si-e ist jedoch sehr wahrscheinlich ein Dativ ${ }^{19}$; seine Bedeutung ist unbekannt und möglicherweise im semantischen Bereich von ,Ruhm“ etc. zu suchen.

(I2) dhal-di-ni-i URU-i-e pu-lu-si,,eine Stele für die (Dat.)/der (Gen.) dem Haldi gehörigen Stadt" HchI $79=$ UKN 30I:I.

19 badusi erscheint nur sehr selten mit der einfachen Auslautschreibung -si; besonders häufig ist die Schreibung -si-e, fast so oft belegt ist -si-i-e (cf. UKN p. 392). Vergleicht man die ebenfalls auf -si/e endenden Wörter alusi/e, pulusi/e, susi/e, die sehr oft im Nominativ belegt sind, so zeigt sich, daß die Graphik -si-i-e hier überhaupt nicht zu finden ist (su-si-i-e UKN 405 ist ergänzt). Die Graphik -Ci-i-e dient aber vor allem in den älteren Inschriften zur Darstellung des Dativs sg. Als Dativ ist $b$. von A. Goetze, RHA 3, fasc. 22 (1936) I83 n. I6; M. de Tseretheli, RA 30 (I933) 3Isq.; F. W. König, HchI p. I77 und Benedict, UPM I62 aufgefaßt worden, während man sonst in $b$. ein Adjektiv mit der Bedeutung ,,großartig, majestätisch, prachtvoll" etc. gesehen hat; cf. M. Salvini, SMEA 9 (I969) 2 Isq. mit Literatur.

Als Beweis für die Deutung als Adjektiv ist von P. Hulin, AnSt 8 (1958) 244 n. 26 , die Form $b a-d u$-si-i-e MEš in einem ebenda veröffentlichten Text gewertet worden (,unless MEš appears on the stone in error"), da der betreffende Satz einen pluralischen Nominativ ( ${ }^{h} h a l-d i-n i$ - $l i \mathrm{~K} \hat{A}^{\mathrm{MES}}$ ) enthält. Dagegen ist einzuwenden, daß $b$. an allen Stellen, wo es sich - bei Interpretation als Adjektiv - auf ein Nomen im Plural bezieht, nie Kongruenz mit diesem aufweist. Falls Adjektive außer den -hi-Bildungen nicht mit ihrem Bezugswort kongruierten, würde dies den Beleg $b a-d u$-si-i-e MEŠ für die adjektivische Deutung entwerten. Ist aber Kongruenz erforderlich, so sprechen die singularischen Belege von $b$. im Zusammenhang mit einem Nomen im Plural gegen die Deutung als Adjektiv. So lange sich also die Verteilung zwischen den Formen von $b$. mit und ohne Meš in Zusammenhang mit einem pluralischen Nomen nicht ändert, wird man mit der von Hulin beigebrachten Form nicht argumentieren können.

Die Interpretation als Adjektiv kann sich auch nicht darauf stützen, daß das Suffix / usi/ ausschließlich adjektivierend sei, da mit urišhusi ,,Magazin" ein klares Substantiv vorliegt. Das zugrundeliegende Wort badi ist zwar belegt, aber semantisch unklar.

Gegen die Deutung als Adjektiv spricht schließlich die Stellung im Satz, insofern als $b$. keineswegs immer vor oder nach dem - bei adjektivischer Deutung - Bezugswort steht, sondern von diesem nicht selten durch das Verb getrennt ist. M. Salvini, SMEA 9 (1969) 22, hat diese Schwierigkeit damit zu beheben versucht, daß er $b$. in letzterem Falle adverbial auffaßt. 
(I3) dḩal-di-ni-ni al-su-i-ši-ni, ,durch die dem Haldi eigene Größe" passim.

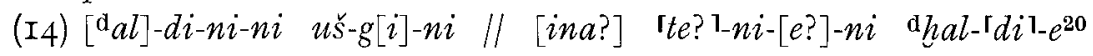
,durch die dem Haldi eigene Gunst" HchI 9 § Io = UKN I9 (Kelišin) 20//I7.

(I5) [ahal-d]i-ni-ni ba-í-ši-i-ni, ,auf Befehl des Haldi“" HchI I22 $\S 4=\mathrm{UKN} 264$ (Topzawä) $\mathrm{I}^{21}$.

Hier schließt Friedrich dann auch die Belege des Typs Țúpa(ni)-naue DINGIR an; er übersetzt ,den zur Stadt Ṭušpa(n) gehörigen Göttern“ und segmentiert also implizit $\operatorname{tus} p a(n)=i n(i)=a u e$, wobei /aue/ als Morphem des Dativs pl.aufgefaßt wird; zu diesen Formen cf. unten II7.

Konfrontiert man diesen Befund mit der Aussage D'jakonovs, das adjektivierende Suffix /ini/ stehe mit dem Genitivsuffix in freier Varianz, kommt man zu folgendem abweichenden Ergebnis:

I. Das Genitivmorphem $|i| \sim|e| \sim|e i|$ steht nur dann, wenn das Bezugswort im Nominativ (d.h. Kasus des Ziels der Handlung im transitiven Satz und des Ausgangs der Handlung im intransitiven) steht.

2. Das Zugehörigkeitssuffix /ini/ steht nur dann, wenn das Bezugswort nicht im Nominativ steht.

Diese Verteilung ist bereits von Gamkrelidze ${ }^{22}$ und Melikišvilii ${ }^{23}$ erkannt worden, doch führt letzterer ohne weitere Diskussion scheinbar widersprechende Belege an: $\mathrm{m}_{\text {menua }}=$ ini hubi "Menua-Tal(?)" oder ,dem Menua gehöriges Tal(?)“ und $\mathbf{m}_{\text {rusa }}=i$ ini hubi „Tal(?), das dem Rusa gehört“"24. Die Sätze, aus denen die beiden Belege entnommen sind, lauten jedoch folgendermaßen:

${ }^{20}$ Cf. W. C. Benedict, JAOS 8r (I96I) 372:20//362: I 7 und Kommentar 367 .

${ }^{21}$ Die assyrische Fassung ist hier nicht ganz klar. M. von Tseretheli, Die neuen chaldischen Inschriften König Sardurs von Urartu, Sitzungsberichte der Heidelberger Akademie der Wissenschaften, Phil.-hist. Kl., Jg. 1927/28, Abh. 5, Heidelberg 1928, und im Anschluß daran J. Friedrich, Caucasica 8 (I93I) I30, lesen mit Interpolation $i-n a\langle q i-\rangle b i-i t$ dhal-di-a; so auch HchI p. I 45. Melikišvili, UKN p. 324, assyr. Fassung I6, liest stattdessen [i]-na a-ma-at im Anschluß an M. de Tseretheli, RA 44 (1950) 190. Die Autographie von C.F. Lehmann(-Haupt), ZDMG $5^{8}$ (1904) 834, gibt Zle. I5 i-na bi-Iit 1 .

${ }^{22}$ VDI 1956/4, I 40. ${ }^{23}$ Melikišvili, US 75.

${ }^{24}$ Melikišvili, US 32 . In seiner Übersetzung der Texte (UKN p. 176,345 ) gibt Melikišvili die Stellen richtig nicht als Nominative wieder: do dolinj(?) Menua ,bis zu dem Tal(?) des Menua“; v ètoj doline(?) (carja) Rusa ,,in diesem Tal(?) des (Königs) Rusa“. 


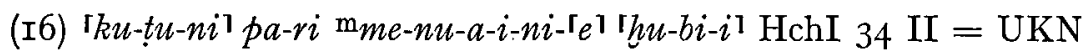
59:5-6. hubi ist kein Nominativ, da die Präposition pari in der Regel den Dativ fordert. Die Übersetzung lautet: „Er rückte vor bis zum Tal(?) des Menua."

(I7) Iil-nu-ka-hi-ni-e $\mathrm{m}_{r u-s a-i-n i-e}$ hu-bi-gi HchI I26 III = UKN 28r:I6- I7. hubigi ${ }^{25}$ ist zweifellos eine Genitivform; zu übersetzen ist: „des betreffenden Tales(?) des Rusa“.

Der von Melikišvili für Kongruenz im Stammkasus ${ }^{26}$ herangezogene Beleg dhaldine DuB-te ist anscheinend aus dem bereits zitierten Satz (II) verkürzt, wo d] hal-di-ni-e ein mit dem folgenden $b a$-du-si-e kongruenter Dativ ist. Auch diese Stelle ist also kein Beleg für /ini/ an einem Nomen mit Bezugswort im Nominativ.

Die häufig in verschiedenen Schreibungen wiederkehrende Formel (I8) haldi kuruni haldini šuri kuruni

ist unterschiedlich interpretiert worden. Man hat haldi als Genitiv aufgefaßt, der von dem als Ablativ-Instrumentalis verstandenen kuruni abhängig sei, und übersetzt: „, durch die Schreckensmacht

${ }^{25}$ Das Zeichen $g i$ ist - m. E. zu Unrecht - von Melikišvili, UKN p. 344 von dem vorausgehenden $h u-b i$ getrennt und als Indefinitpronomen (sonst meist $g i-e-i)$ aufgefaßt worden. Die auf $g i$ folgende Konjunktion aše ,,wenn" steht normalerweise am Satzanfang. Wenn diese Position nicht zwingend ist, könnte allerdings die Satzgrenze hinter dem Wort tini liegen; der folgende Satz wäre dann zu übersetzen: ,Wenn der Kanal des betreffenden Tales(?) des Rusa überläuft(?), soll man dem Haldi ein Böckchen schlachten." Cf. Diakonoff, HuU II7 n. I26. Anderenfalls ist zu übersetzen: „Umeši ist der Name des betreffenden Tales(?) des Rusa."

$h u$-bi-gi enthält den ,Hiatustilger" -g-; cf. Melikišvili, US 22sq.; Friedrich, Urartäisch 37. Die Aussprache dürfte je nach dem Grad der Geschlossenheit der benachbarten Vokale zwischen [j] und $[\gamma]$ wechseln. Diakonoff, $\mathrm{HuU} 5$, schlägt vor, für GA, GI, GU in vielen Fällen die Lesung $j a_{\mathbf{x}}, j e_{\mathbf{x}}, j u_{\mathbf{x}}$ einzuführen. Der ,Hiatustilger" erscheint auch vor dem Genitiv- und dem Dativsuffix; cf. Melikišvili, US 22. Vielleicht sollte man das Genitiv- und das Dativmorphem grundsätzlich als $/(y) i / \mathrm{bzw} . /(y) e /$ ansetzen, wofür auch die vor allem in älteren Texten häufigen plene-Schreibungen $-e-i$ bzw. -i-e ein Indiz sein könnten. Dann wäre auch der etymologische Zusammenhang mit den entsprechenden hurritischen Suffixen /ve/ und /va/ unproblematisch; letztere werden unter bestimmten Bedingungen bilabialisiert ( $>/$ ue/ bzw. $/ u a /)$, und /ve/ erscheint in bestimmten Positionen als /ye/; cf. Bush, GHL 9osqq. Der Vokal des Genitivmorphems ist im Hurritischen der BoğazköyTexte geschlossener als $e$ gesprochen worden, wie die Schreibungen mit $w i_{i}$ neben $w e_{e}$ zeigen. Dasselbe Schwanken zwischen $e$ und $i$ in der Darstellung des Genitiv-Morphems zeigt auch das Urartäische; cf. Friedrich, Urartäisch 37 II, $39 \S 23$.

${ }^{26}$ Melikišvili, US 74 . 
bei Hyaldi ..."27. Das auslautende -ni von kuruni kann jedoch nicht das Abl.-Suffix sein, da gelegentlich kurunini belegt ist. Andere haben in kuruni ein Adjektiv im Nominativ gesehen ${ }^{28}$ und übersetzt: "Haldi ist mächtig, die Waffe ${ }^{29}$ des Haldi ist mächtig." Ist dies richtig, so müßte suri als Nominativ aufgefaßt werden, so daß die darauf bezogene Form haldini im Gegensatz zu der oben aufgestellten Verteilungsregel stünde.

Die verschiedenen Varianten zeigen, daß ein Suffix /ni/ fakultativ an jedes Wort des Satzes (I8) treten kann. Dies wie auch die Unsicherheit hinsichtlich der Semantik von kuruni macht eine zufriedenstellende Analyse der Formel zur Zeit noch unmöglich.

Es ist keineswegs ausgeschlossen, da $B$ - was $\mathrm{m}$.W. bisher nicht erwogen wurde - kuruni(ni) eine Postposition ist, die sich mit verschiedenen Kasus (wie vergleichsweise die Präposition pari) verbindet. Hierfür sprechen zwei Belege: dhal-di-i ku-r[u-ni DIN]GIR MEŚs-na ku-ru-

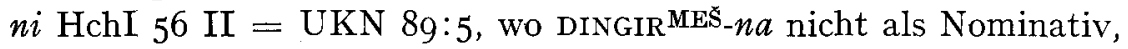
sondern nur als Lokativ pl. aufgefaßt werden kann. Ebenso $\mathrm{KUR} b a$ ba-ni-a[k]u-ru-ni-e HchI II8 II = UKN 266:I2-13, wo ein Lokativ sg. vorliegt. Eine Bedeutung ,mit, auf/an der Seite" würde allen Belegen von kuruni(ni) durchaus gerecht. Auch die Stelle HchI 7 $\mathrm{III}=\mathrm{UKN} 24$ lic. $\mathrm{I}_{3}-\mathrm{I} 4[m a]-a-n u \mathrm{~m}_{i s ̌}-[p u]-u-i-n i k u-r u-n i \mathrm{~m}_{m e-n u-}$ $\dot{u}-a k u-v w$ - $\left[n i\right.$ LÚ] $h u-v a-d i-n a-a$ I ME 6 GIS̆ GIGIR $^{1} \ldots$ müßte dann nicht - wie bei adjektivischer Interpretation von kuruni notwendig in zwei Sätze zerlegt werden, wobei der zweite ein Nominalsatz ohne Kopula(?) / ni/ (cf. unten n. 36) wäre. Stattdessen könnte man sinnvoll übersetzen: ,,Es war(en) mit/auf der Seite von Išpuini (und) mit/auf der Seite von Menua im Heer Ioo6 Streitwagen, ....“

Läßt man diese Deutung gelten, könnte hal-di-ni šu-ri-i ein von kuruni abhängiger Genitiv sein. Doch vorläufig wird man besser die problematische Formel in dem hier behandelten Zusammenhang außer Betracht lassen.

Eine Durchsicht aller urartäischen Inschriften hat keinen sicheren Beleg ergeben, der gegen die oben festgestellte Verteilung der Formen

${ }^{27}$ F. W. König, HchI p. I9I b; Friedrich, Urartäisch 52; mit Bedenken Benedict, UPM I23sq.

28 So Friedrich, Einführung 49 (,stark"??); idem, Acta Jutlandica 9 (I937)

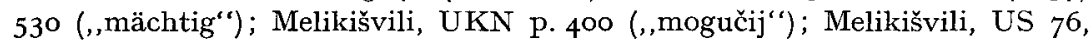
84 (,,mächtig", , ,kräftig", , ,gewaltig", ,,hoch" [Berg]).

${ }^{29}$ Zur Bedeutung "Waffe" von GIŠšuri/e cf. Benedict, UPM I23 n. 9 mit Lit. Neuerdings ist urart. šuri/e mit hurr. šauri „Waffe" in einen etymologischen Zusammenhang gebracht worden; cf. V. Haas, OrAnt I I (I973) 233. 
mit /ini/ und denen mit Genitivsuffix auf syntaktisch unterschiedliche Positionen spricht.

Die grammatische Analyse des als / ini/ morphologisierten Segments ergibt sich am deutlichsten aus der Gegenüberstellung zweier kurzer Inventarbezeichnungen, die in identischer Struktur bei wechselndem Königsnamen in mehreren Exemplaren erhalten sind ${ }^{30}$ :

(I9) $\mathrm{m}_{m e-n u-a-i} \dot{u}-r i-i[\check{s}]-\not h i$,Gerät des Menua“ HchI $40 \mathrm{~B}=$ $\mathrm{UKN}$ II8a.

Dem Nominativ urišhi ist hier der Besitzer im Genitiv vorangestellt. In der zweiten Inschrift dagegen steht das übergeordnete Nomen, ein / usi/-Derivat von urišhi mit der Bedeutung "Magazin“ (gelegentlich mit É „Haus“ determiniert, das vereinzelt auch, wohl versehentlich, auf urišhi übertragen wurde) im Genitiv nach Artikel sg. :

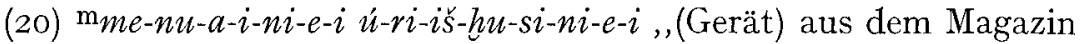
des Menua" HchI $40 \mathrm{~A}=\mathrm{UKN}$ II2-II7.

In der kurzen Inschrift auf dem urartäischen Kandelaber in Hamburg ${ }^{\mathbf{3 1}}$ stehen beide Syntagmata nebeneinander:

(2I) $\mathrm{m}_{r u-s a-a-i}$ ta-na-a-și $\mathrm{m}[r] u-s a-i-n i-i$ u-ri-iš-hu-si-ni-i , Leuchter des Rusa, aus dem Magazin des Rusa."

Meine Erklärung des Befundes greift auf ein gleichfalls von Friedrich festgestelltes Morphem zurück, nämlich das sogenannte ,,suffixanreihende" $/$ ni/32. Dieses Morphem ist am besten aus der ,Suffixaufnahme" am Patronymikon nach Ergativ bekannt:

(22) $\mathrm{m}_{m e-i-n u-\imath-a-\check{s} e} \mathrm{~m}_{i \breve{s}-p u-u-i-n i-h i-n i-s ̌ e}$,Menua, der Sohn des Išpuini“" HchI $69 \mathrm{I}=\mathrm{UKN}$ I07:4-5//I8-I9 und ähnlich passim.

Neuere Darstellungen der urartäischen Grammatik haben diese Erkenntnis Friedrichs leider wieder aufgegeben ${ }^{33}$. So setzt Melikišvili

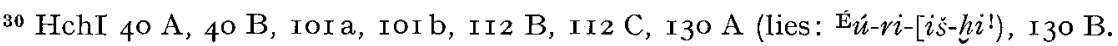
Die Texte entsprechen (in der gleichen Reihenfolge): UKN I I 2-II7, I 8 a, I 5O, I 5 I; 262-263, I 77- I90, 283, 270-274d.

31 J. Friedrich, Der urartäische Kandelaber in Hamburg und seine Keilinschrift, 2. Die Keilinschriften des urartäischen Kandelabers, ZDMG III (I96I) $285-287$, Tf. I, III.

32 J. Friedrich, Zum Subaräischen und Urartäischen, Fs. Deimel, AnOr I2, Rom I935, I27sq.; idem, Kleine Beiträge zur churritischen Grammatik, MVAeG 42/2, Leipzig I939, 6I.

${ }^{33}$ Nur M. Salvini spricht neuerdings wieder von einem ,,suffixanreihenden Element" /ni/; cf. Anhang zu Melikišvili, US 97. 
ein Zugehörigkeitssuffix /hi(ni)/ $\mathrm{an}^{34}$ und unterstellt damit freie Varianz von /hini/ und / hil. Doch auch hier gilt die gleiche Verteilung, wie sie oben für das Genitivmorphem und das angebliche Zugehörigkeitssuffix /ini/ festgestellt wurde:

I. |hil steht nur, wenn das übergeordnete Nomen im Nominativ steht; z.B. $\mathrm{m}_{\text {rusa }}=n i \mathrm{~m}_{\text {argi }}$ šti=hi ,,Rusa, der Sohn des Argišti".

2. /hini/ steht bei allen anderen Kasus des Singulars (zum Plural cf. unten); z. B. das oben für den Ergativ angeführte Beispiel sowie die beiden folgenden für den Genitiv und den Dativ: $\mathrm{m}_{r u s a}=i \mathrm{~m}_{\text {argišti }}=$ hini $i \mathrm{HchI}$ I03 $\mathrm{A}=\mathrm{UKN} 283 ; \mathrm{m}_{\text {isppuini=e }} \mathrm{m}_{\text {sarduri }}$ hini=e $\mathrm{HchI}$ $\mathrm{I} 2=\mathrm{UKN}$ I8:I3-I4 (weitgehend, aber sicher ergänzt).

D'jakonov hat diese Verteilung bereits beobachtet ${ }^{35}$; er identifiziert /ni/ zu Recht mit dem Artikel sg. des Hurritischen /ne/, verkennt aber die Struktur der Opposition rusa=ni argišt $i=h i$ gegen $r u s a=i$ argišt $i=$ $h i=n i=i$, wenn er die beiden Syntagmata dahingehend interpretiert, da $B$ eine Konstruktion mit adjektivischem Attribut auf |hil den Artikel nur einmal enthalte, im ersten Falle bei dem durch das Adjektiv bestimmten Personennamen, im zweiten Falle beim Adjektiv.

Auf das Suffix / $n i /$ im ersten Syntagma kann hier nicht eingegangen werden; ich möchte es jedoch nicht mit dem Artikel sg. identifizieren, sondern es mit dem enklitischen Personalpronomen der 3. Ps. sg. des Hurritischen $|n / \sim / n n a|^{36}$ etymologisch verknüpfen.

${ }^{34}$ Melikišvili, US 31 ; ebenso Benedict, UPM I 26sq.

${ }^{35}$ Diakonoff, HuU Ioo n. Io6; cf. auch T. V. Gamkrelidze, VDI 1956/4, I40. Die Einwendungen von Benedict, UPM I27 n. I7 unter Hinweis auf die Konstruktionen mit urišshi und urišhusi=ni sind unberechtigt; der einzige widersprechende Beleg beruht auf einer Ergänzung (Benedict, UPM I26); zu dieser cf. oben n. 30 .

${ }^{36}$ Das enklitische Personalpronomen der 3. Ps. sg. zur Bezeichnung des Ausgangs der Handlung im nichtergativischen und des Ziels der Handlung im ergativischen Satz ist von A. Goetze, JCS 2 (I948) 259sqq., bestimmt worden, der auch eine Kurzform $/ n /$ beobachtet hat. W. Farber, Or 40 (I97I) 29-66, hat für alle enklitischen Pronomina funktional identische Kurzformen festgestellt $(|d \sim| t t a|| d i, l / \sim \mid$ dilla $|| l,|\sim| l l a /)$ und mit der Kurzform $\mid n /$ auch die gleichlautende sogenannte , Kopula“ identifiziert, ohne diese als syntaktische Kategorie aufgeben $\mathrm{zu}$ wollen (p. 43).

Das urartäische Suffix / ni/ am Ausgang der Handlung in nichtergativischen Sätzen ist als ursprünglicher Artikel aufgefaßt worden, der in Entwicklung ",halbflektierender Systeme", zu denen die nicht mehr klar abzusondernden Suffixpositionen verschmolzen seien, Kennzeichen der ,determinierten Deklination" geworden sei; cf. Diakonoff, HuU 89. Ein umfangreiches Beleg- 
Entgegen D'jakonovs Auffassung existiert für das zweite Syntagma durchaus eine exakte Parallele im Hurritischen ${ }^{37}$, nur ist dabei ebenso wie im Urartäischen Voraussetzung, daß das übergeordnete Nomen nicht im Nominativ steht ${ }^{38}$ :

material für die ,determinierte Deklination" kann allerdings nur für den Nominativ geboten werden. Friedrich hat bereits erwogen, das Suffix /ni/ im Nominativ mit der hurritischen Kopula zu identifizieren; cf. Friedrich, Urartäisch 47. Bei dieser Interpretation des Suffixes entfiele auch die auf dem Hintergrund des Hurritischen störende häufige Determinierung der Personennamen (im Mit. ist ein Personenname nie durch den Artikel / ne/ determiniert). Auch die Tatsache, daß bei zweigliedrigem Ausgang der Handlung das Element / $n i$ / gelegentlich nur einmal steht (so in der KelišinBilingue 4 und 27; išpuini=ni ... menua ,I. und M.“, cf. W. C. Benedict, JAOS 8I (I96I) 373 ad 4), könnte ein Reflex der ursprünglichen positionalen Mobilität der Kopula bzw. der enklitischen Personalpronomina sein.

Zur Beurteilung der Form des urartäischen Suffixes gegenüber der abweichenden Form im Hurritischen des Mit. ist $z u$ berücksichtigen, daß im Boğazköy-Hurritischen dem $/ n / \sim / n n a /$ des Mit. wahrscheinlich wenigstens gelegentlich /ni/ entspricht; cf. V. Haas/G. Wilhelm, AOAT-S 3, Kevelaer/ Neukirchen-VIuyn I974, 134 .

Der urartäische Artikel sg., auf dem nach D'jakonov die ,determinierte Deklination" basiert, ist in nicht-korrelativer Funktion nur in verhältnismäßig wenigen Beispielen zu belegen (cf. Diakonoff, $\mathrm{HuU} 9 \mathrm{I}$, wo die meisten Belege im Sinne der in diesem Aufsatz dargestellten Konstruktion des Attributs interpretiert werden müssen. $Z u$ nennen wären aber in diesem Zusammenhang die Bildungen biai=ni=še und lului=ni=še ,ein Urartäer" bzw. ,ein Ausländer", die beide von $i$-stämmigen Adjektiven abgeleitet sind; cf. lulue ,,wild, unkultiviert" sowie die Pluralbildung der Landesnamen biai=ni=li, lului=ni=li, die wegen ihrer Parallelität mit Toponymika der adjektivischen Basis Königsname $=\underline{h} i=$ daraufhinweist, daß Adjektiva zugrundeliegen).

Daß der Artikel, die Kopula und das enklitische Personalpronomen der 3. Ps. sg. ebenso wie gewisse Demonstrativpronomina (urart. ina-, hurr. anni, anu-) voreinzelsprachlich im Sinne eines pronominal-deiktischen Elements identisch sind, kann als sehr wahrscheinlich angenommen werden, darf aber natürlich nicht dazu verleiten, die einzelsprachlichen Differenzierungen zu ignorieren. Es ist auch nicht möglich, die verschiedenen /ni/-Suffixe mit T. V. Gamkrelidze, VDI $1956 / 4,138$, und Melikišvili, US 34 n. 55, auf das Demonstrativpronomen ini zurückzuführen; der Stamm dieses Pronomens ist als $i$ - anzusetzen (dazu Nom. sg.: $i=n i$, Nom. pl.: $i=n i=l i$, Abl.-Instr. pl.: $i=n a=n i$, Lok. pl.: $i=n a=a)$; es steht vor oder nach seinem Bezugswort und ist von dem Demonstrativpronomen ina-, das stets voransteht, zu trennen (zu diesem Nom. sg.: ina=ni, Nom. pl.: ina $=n i=l i$, Abl.-Instr. pl.: ina $=n a=n i$ [unv.]).

${ }^{37}$ Diakonoff, HuU ıоo n. เо6.

${ }^{38}$ So das von $D^{\prime}$ jakanov, l.c. angegebene Beispiel tugris̄ $=h$ e evrni ,,der König von Tugrižz" KUB XXVII 38 Rs. IV I4. 
(23) hur-wu-u-hé-ni-e-we KUR $u-u-\langle m i-\rangle i-i n-n i-e-w e$ (hurv=o=ǵg=ne=ve omini $=n e=v e)$, des hurritischen Landes" Mit. II 72.

Wenn man von dem beim urartäischen Personennamen fehlenden Artikel absieht, ist die strukturelle Übereinstimmung vollkommen. Der Artikel hat in solchen Syntagmata eine korrelative Funktion ${ }^{39}$;

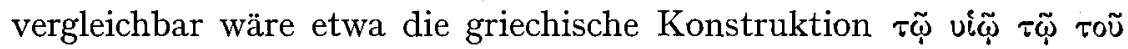

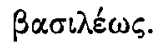

Der gleichen Konstruktion mit korrelativem Artikel und Suffixaufnahme unterliegen im Hurritischen auch Genitivattribute ${ }^{40}$, wobei der korrelative Artikel nur dann suffigiert wird, wenn das Bezugswort nicht im Nominativ steht, der ja seinerseits auch suffixlos ist. Ein Genitivattribut kongruiert also im Hurritischen, anders als etwa im Deutschen, mit seinem Bezugswort in Kasus und Numerus:

(24) še-e-ni-iw-[wu]-ú-e-ni-e-we KUR $u$-u-[m]i-i-ni-i-we $(\tilde{s} e n(a)=i f f u=$ $u e=n e=v e$ omini=ve) , des Landes meines Bruders" Mit. I 97 (Genitiv)

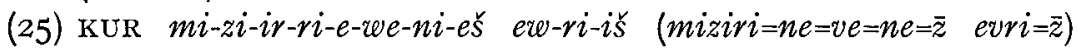
„der Herr Ägyptens“ Mit. I 85 (Ergativ)

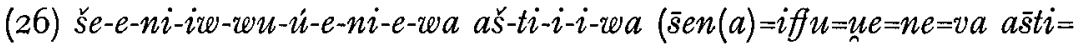
$(y i=) v a)$, der Gattin meines Bruders" Mit. II 6 (Dativ)

(27) ร̌e-e-ni-iw-wu-ú-e-ni-e-en-nu-uh-ha ti-ša-a-an-nu-uh-ha $(\bar{s} e n(a)=$ iff $u=u e=n e=n n(i)=0=h h(e)=a$ ti $\bar{z} a=n n(i)=0=h h(e)=a)$, in der Herzlichkeit meines Bruders (?)“ Mit. II Io.

In derselben Weise sind auch die urartäischen Formen mit einem fälschlich als / ini/ segmentierten Element aufzufassen; es sind Genitivformen ${ }^{41}$, die nach dem in korrelativer Funktion verwendeten Artikel die Kasusendungen des Bezugsworts aufnehmen und dadurch mit diesem Kongruenz herstellen.

${ }^{39}$ Diakonoff, $\mathrm{HuU}$ roo.

${ }^{40}$ Bush, GHL I $49-$ I 53, I $_{57}-\mathrm{I} 59$.

41 Bei $a$ - und $u$-Stämmen schwindet das Genitivmorphem in dieser Position nicht selten; cf. DINGIR ${ }^{\mathrm{MES}}$ atqana $=* i=n a=u e \mathrm{HchI}$ Io II $19 / / \mathrm{X}{ }_{4}=\mathrm{UKN}$ 27:19//64 versus $\mathrm{d} u a=i=n a=u e$ sešti=na=ue $\mathrm{HchI}$ Iо II $20 / / \mathrm{X} 66=\mathrm{UKN}$ 27:20//66; URU qumenu $={ }^{*} i=n a=u e \mathrm{HchI}$ Iо II I4//X $55=\mathrm{UKN}$ 27:14//55 versus [UR] $[b a b] i l u=i=n e=e$ KURebani=edi $\mathrm{HchI} 80 \S 5 \mathrm{~V}=\mathrm{UKN}$ I27 III I I (in letzterem Beispiel wirkt der ursprünglich postpositionale Charakter des Direktivsuffixes in der Weise nach, daß das Attribut nicht gleichfalls das Direktivsuffix trägt, sondern in dem Kasus steht, der von der ursprünglichen Postposition gefordert wird, nämlich im Dativ; cf. edia mit vorangehendem archaischem Dativ sg. / $/ \mathrm{e} e /)$. 
Die oben in syllabischer Umschrift wiedergegebenen Syntagmata (ro)-(I5) mit angeblichem /ini/ stellen sich in analytischer Transkription demnach nun folgendermaßen $\mathrm{dar}^{42}$ :

(Io) menua $=i=n e=i$ sil $a=i$,,der Tochter (Gen.) des Menua“"

(II) haldi $=i=n e=e$ badusi=e „für den Ruhm (?) des Haldi“

(I2) hald $i=i=n e=e$ patar $i=e$ pulusi , eine Stele für die Stadt des Haldi“

(I3) haldi=i=ne=ni alsuiši=ni, ,durch die Größe (Instrumentalis) des Haldi"

(I4) ald $i=i=n e=n i u s ̌ g i=n i$,,durch die Gunst des Haldi“

(×5) hald $i=i=n e=n i$ bauš $i=n i$, auf Befehl des Haldi“

A. Götze hat I930 in seiner Bearbeitung der Kelišin-Stele aufgrund der Form haldina(ni) kÁ sowie vieler Formen des Genitivs pl. auf -naue ein Pluralmorphem /na/ angesetzt ${ }^{43}$, ohne daß ihm die weitere Forschung darin gefolgt wäre. Völlig in die Irre ging die Kritik C. F. Lehmann-Haupts ${ }^{44}$, während J. Friedrichs Bestimmung eines Pluralmorphems $/\left.a\right|^{45}$ bis heute in alle Darstellungen der urartäischen Grammatik Eingang gefunden hat ${ }^{\mathbf{4 6}}$. Nur M. Salvini hat neuerdings wieder in Erwägung gezogen, $|n a|$ als Pluralzeichen bei obliquen Kasus anzusetzen ${ }^{47}$.

Das in vielen Fällen vorausgehende $n$ betrachtet Friedrich als meist zu dem Zugehörigkeitssuffix / ini/gehörig, wobei der auslautende Vokal vor dem vokalischen Pluralzeichen ausgefallen sei. G. A. Melikišvili sieht eine Tendenz, die Kasusendungen außer der des Nominativs im Plural nicht an den reinen Stamm, sondern an den Nominativ anzufügen ${ }^{\mathbf{4 8}}$, worunter er den reinen Stamm plus /ni/-Suffix versteht. Auch D'jakonov nimmt $|a|$ als Zeichen des Plurals in obliquen Kasus an, betrachtet aber das vorausgehende - $n$ - als den Artikel, mit dem die sog. ,determinierte Deklination“" gebildet werde ${ }^{\mathbf{4 9}}$.

42 Der relationale Artikel wird zur Verdeutlichung wie im Hurritischen mit /ne/ wiedergegeben, obwohl der urartäische Befund hinsichtlich der Qualität des Vokals nicht klar ist. Ebenso ist das Genitivmorphem immer als $/ i$, das Dativmorphem immer als $/ e /$ wiedergegeben.

43 A. Götze, ZA 39 (I930) I I 5.

${ }^{44}$ C. F. Lehmann-Haupt, Klio 24 (I93I) I56sqq.

$45 \mathrm{~J}$. Friedrich, $\mathrm{ZA}_{4} \mathrm{O}$ (I93I) 274.

${ }^{46}$ Melikišvili, US $3^{8}$; cf. auch 40, 4I; Benedict, UPM I 76 ; Friedrich hat merkwürdigerweise weder in seiner Einführung ins Urartäische noch in deren Überarbeitung (Friedrich, Urartäisch) ausdrücklich auf das von ihm selbst etablierte Pluralmorphem $/ a /$ hingewiesen.

47 M. Salvini, ZA 6I (I97I) 253 n. 24.

48 Melikišvili, US 35.

49 Diakonoff, HuU 96. 
Der Ansatz eines Pluralzeichens /a/ scheint dadurch bestätigt $\mathrm{zu}$ werden, daß in manchen Formen vor $|a|$ ein anderer Konsonant als $n$ steht:

šuraune im Titel „König der šuri-Länder“.

evelaue im Titel , König der Könige“"

[p]ilaue Dativ pl. von pili „Kanal“ HchI I2I I = UKN 268:3.

$[d]$ iraue Dativ pl. ([d] $i$ unsicher) $\mathrm{HchI}$ Io II I9 $/ / \mathrm{X} 65=$ UKN 27:I9//65.

Alle Pluralformen ohne $n$ vor dem angeblichen Pluralmorphem */a| lassen sich zwanglos durch Annahme eines im Hurritischen wohlbezeugten Lautgesetzes in der Weise erklären, daß hier die Lautfolge rin $>r(r)$ und $l$ in $>l(l)$ geworden sei. Ebenso wie hurr. avarre als

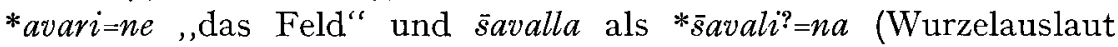
nicht gesichert) ,die Jahre" zu analysieren sind, ist dann urart. šuraue auf $*$ suri=na=une, erelaue auf $*$ ereli=na=ue etc. zurückzuführen.

Anders als im Hurritischen gilt dieses Lautgesetz jedoch nicht für die Lautfolge nin (hurr. enna $<*$ eni=na, ominne $<*$ omini=ne, aber urart. ardini=na=ue etc.). Falls artu'ar(a)saue $\mathrm{HchI}$ Io II I4//X $54=$ UKN 27:I4//54 als Dativ pl. aufzufassen ist, müBte man im Urartäischen zusätzlich mit $s(i) n>s(s)$ rechnen, wofür im Hurritischen immerhin eine Analogie in der Assimilation des enklitischen Personalpronomens der 3. Ps. sg. an das vorhergehende Ergativmorphem $\left({ }^{*} \bar{z}=n n a>\bar{s} \bar{s} a\right)^{50}$ beizubringen wäre. Sehr wahrscheinlich handelt es sich aber um den Dativ sg. eines u-Stamms.

Die etymologische Verknüpfung mit dem gleichlautenden Artikel pl. des Hurritischen liegt auf der Hand und läßt sich noch durch die in beiden Sprachen übereinstimmende Verwendung von/na/als Suffixrelator am Attribut bei pluralischem Bezugswort erhärten:

(28) dhal-di-na-i-e BE-LI MES (haldi=i=na=ue $B \bar{E} L \bar{I}=* u e)$, den Waffen des Haldi" HchI Io II 7//X 4I = UKN 27:7//4I.

(29) dhal-di-na-u-e KÁ (haldi=i=na=ue *ššti=na=ue) , den Toren des Haldi" HchI Io II I6//X $58=\mathrm{UKN}_{27}: \mathrm{I6} / / 5^{8}$.

(30) $\left.\mathrm{KUR}_{e}-b a-n i-n[a-i]\right]-e$ DINGIR (ebani=i=na=ue DINGIR=na=ue oder ebani=na=ue DINGIR=e) ,,den Göttern des Landes“ oder ,,dem Gott der Länder"51 HchI Io II I8//X $63=$ UKN 27: $8 / / 63$.

${ }^{50}$ W. Farber, Or 40 (197I) 32-4I.

51 Die große Opfervorschrift vom Meher kapısı (HchI ıо $=$ UKN 27) enthält

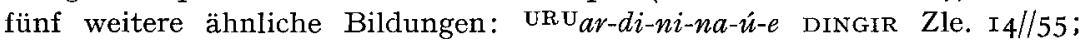
URU $q u-m e-n u-n a-\hat{u}-e$ DINGIR Zle. I4//55; URU $t u-u s ̌-p a-(n i-) n a-\hat{u}-e$ DINGIR Zle. 
Im übrigen jedoch weicht die Funktion von /na/ im Urartäischen von der im Hurritischen ab:

I. $|n a|$ steht regelmäßig vor allen Kasusendungen außer der des Nominativs im Plural.

2. $|n a|$ hat keine determinierende Funktion im Sinne eines Artikels. Die durch Bezeichnung der Determination bzw. Indetermination hergestellte Opposition hurr. tive=na , die Worte/Dinge“: *tive=az „,Worte/Dinge“ (belegt ist $t u p p i=a \bar{z}$,,Tontafeln“) kennt das Urartäische nicht.

Hinsichtlich der Suffixfolge am Nomen ergibt sich damit für das Urartäische ein ähnlich klares Bild wie für das Hurritische. Nur die Position des hurritischen Pluralzeichens / $a \bar{z} /$ bleibt im Urartäischen von archaischen Bildungen abgesehen ${ }^{52}$ - unbesetzt, da dessen Funktion von dem ursprünglichen Pluralartikel $/ n a \mid$ übernommen wird. Allerdings wissen wir noch nichts über die Pluralbildung der Possessivsuffixe, so daß hier wohl eine Position, nämlich eben die von hurr. $|a \bar{z}|$, reserviert werden muß. Unklar ist ferner, ob im Urartäischen der Artikel zusammen mit den Possessivsuffixen auftreten kann, wie dies im Hurritischen der Fall ist ${ }^{53}$.

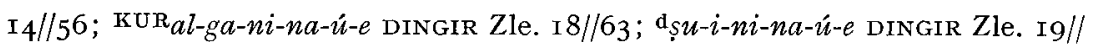
64. Bei allen Belegen folgt kein Pluraldeterminativ auf DINGIR, obwohl zumindest bei den Städtenamen die in den Suffixen $=n a=u e$ ausgedrückte Pluralität sich natürlich nur auf DINGIR, nicht auf die Stadt beziehen kann, während die letztgenannten Stellen auch algani=na=ue DINGIR ,,dem Gott der Gebirge(?)" etc. gedeutet werden könnten. Das Pluraldeterminativ ist im Urartäischen nicht obligatorisch, wie vor allem die Belege für KÁ $\sim$ KÁMEŠ zeigen. In dem hier erörterten Text ist allerdings auffällig, daß dort, wo das Pluraldeterminativ hinter DINGIR steht, der Genitiv folgt (z.B. DINGIR MES̆ atqana $={ }^{*} i=n a=u$ Ze Zle. I9//64), während er in den sechs Fällen, in denen MEš fehlt, voransteht. Für eine Auffassung des DINGIR als Singular spricht die leider nicht sicher zu ergänzende Stelle HchI I02 III = UKN I56: I3, wo das syllabische Komplement den Dativ sg. angibt: I UDU $A N A$ ?? $/\{\mathrm{I}\}$ d $[s u-i-n i ? ?]-i-n a-i-e$ DINGIR-i-e. Ist dieser Beleg - er steht hinsichtlich des Komplements bisher vereinzelt - ernstzunehmen, so würde dies bedeuten, daß bei pluralischem Genitiv keine Suffixaufnahme stattfindet. Für das Hurritische fehlt es im Mit. hierzu an Vergleichsmaterial.

${ }^{52}$ Neben dem Direktiv pl. /naedi/ /nadi/ begegnen Bildungen auf /ašte/, in denen der im Hurritischen außer im Nominativ pl. obligatorische Pluralisator / $a \bar{z} /$ konserviert ist; cf. Diakonoff, $\mathrm{HuU}$ I02 n. 108.

53 Der Artikel steht dabei in der Position $+I$, das Possessivsuffix in +2 ; so mit F. W. Bush, AOAT 22 (1973) 5o gegen A. Kammenhuber, MSS 23 (I968) 50, 58; letztere Darstellung wiederholt in $\mathrm{RlA}_{4}$, 6./7. Lfg., 513, r. Sp. 
Fassen wir die Ergebnisse zusammen:

I. Ein Zugehörigkeitssuffix / ini/ existiert nicht.

2. Ein Pluralzeichen $\mid a /$ existiert nicht.

3. Bei Genitiv- und hi-Attributen werden die Kasussuffixe des Bezugsworts nach dem relationalen Artikel/ne/(sg.) oder /na/ (pl.) angefügt, wenn das Bezugswort in einem obliquen Kasus steht.

4. In allen Kasus des Plurals mit Ausnahme des Nominativs tritt ein Pluralisator /na/ auf, der mit dem Artikel pl. des Hurritischen etymologisch zu verknüpfen ist.

5. Ist der letzte Konsonant eines Nomens $l$ oder $r$, so schwindet der stammauslautende Vokal vor antretendem / na/ unter Assimilation des $n$ an den vorhergehenden Konsonanten, wie dies auch im Hurritischen der Fall ist.

Abkürzungen (außer den üblichen für Serien- und Zeitschriftentitel):

Benedict, UPM = Warren C. Benedict, Urartian Phonology and Morphology, Ph. D. University of Michigan 1958.

Bush, GHL = Frederic William Bush, A Grammar of the Hurrian Language, $\mathrm{Ph}$. D. Brandeis University 1964 .

Diakonoff, $\mathrm{HuU}=\mathrm{I}$. M. Diakonoff, Hurrisch und Urartäisch, MSS Beiheft 6 N. F., München I97I (ïberarbeitete Übersetzung von I. M. D'jakonov, Jazyki drevnej Perednej Azii, Moskva I967, 29sqq., I I3sqq., 443sqq.).

Friedrich, Einführung = Johannes Friedrich, Einführung ins Urartäische, MVAeG 37/3, Leipzig I933.

Friedrich, Urartäisch = Johannes Friedrich, Urartäisch, in: Handbuch der Orientalistik, I. Abt., 2. Bd., I./2. Abschnitt, Lfg. 2, Leiden/Köln I969, $3 \mathrm{I}-53$.

HchI = Friedrich Wilhelm König, Handbuch der chaldischen Inschriften I-II, AfO Beiheft 8, Graz I955, I957.

Melikišvili, US $=$ G. A. Melikišvili, Die urartäische Sprache, mit einem Anhang von Mirjo Salvini, Studia Pohl 7, Rom I97I (Übersetzung von G. A. Melikišvili, Urartskij jazyk, Moskva 1964).

Mit. = Der Mittanni-Brief, zit. nach: Johannes Friedrich, Kleinasiatische Sprachdenkmäler, Berlin I932, 8-32.

$\mathrm{UKN}=\mathrm{G}$. A. Melikišvili, Urartskie klinoobraznye nadpisi, Moskva I960.

M. Salvini sei herzlich für seine Bereitschaft gedankt, die Probleme dieses Aufsatzes brieflich zu diskutieren; er hat, bei grundsätzlicher Zustimmung zu den hier vertretenen Thesen, zahlreiche Anregungen zu Detailfragen gegeben, die dankbar aufgegriffen wurden. 